\title{
A Study on the Interest Factors of "Collaborative Inertia" of Intergovernmental Public Service Provision in China
}

\author{
Xiaoning $\mathrm{Zhu}^{1, *}$, Yanyu Chen ${ }^{2}$ \\ ${ }^{1}$ School of Public Affairs and Administration, Electronic Science and Technology of China, Chengdu, Sichuan \\ 6117311 \\ ${ }^{2}$ School of Public Affairs and Administration, Electronic Science and Technology of China, Chengdu, Sichuan \\ 6117312 \\ *Corresponding author. Email: zhuxn1956@163.com
}

\begin{abstract}
In China, the supply of public services, once limited to administrative districts, has gradually broken through the rigid constraints and become a complex regional public service supply problem. Local governments have begun to change their traditional closed management style and strengthen horizontal ties with other regional governments, and horizontal collaboration among governments in providing public services has become a major trend. As the main supplier of public services, whether local governments are motivated to collaborate and whether they can turn their motivation into action largely determines the quality and efficiency of public service provision. As a kind of collaborative system, the horizontal inter-governmental public service supply also suffers from "collaborative inertia", i.e., weakened motivation, low efficiency and obvious conflicts. From the perspective of the root cause of "collaborative inertia", the interest factor plays a key role in the collaborative supply among local governments. This paper analyzes how the key factor of interest acts on the motive of synergistic supply, constructs a three-dimensional analysis model of "collaborative inertia", analyzes the inner movement mechanism of each dimension, and proposes measures to alleviate "collaborative inertia", providing theoretical suggestions for realizing the synergistic supply of intergovernmental public services, and providing decision-making reference for the practice of synergistic supply of intergovernmental public services.
\end{abstract}

Keywords: Intergovernmental Cooperation, Public Service Supply, Cooperative inertia, Interest Factor

\section{INTRODUCTION}

Intergovernmental relations refer to the intertwined network of relationships between the central government and local governments at all levels. The inter-governmental relationship studied in this paper focuses on horizontal inter-governmental relationship, i.e., the relationship between local governments at the same level without administrative affiliation and local governments. Collaborative horizontal inter-prefectural public service provision is a process in which local governments and local governments cooperate with each other to share power and jointly provide public goods and services in an interdependent environment in order to satisfy public access and achieve efficient supply of quality public services and products across administrative regions.
Local governments are an important part of the overall national administrative system, and the provision of cross-regional public services is one of the purposes of horizontal cooperation among local governments. In recent years, the concept of "cross-regional services" in public service provision has gradually taken root in various regions, and the horizontal inter-governmental cooperation across administrative regions has been gradually strengthened, which has played a positive role in changing the image of government, improving the quality of public service provision, increasing the efficiency of public service provision, and reducing the cost of public service provision.

At present, in the face of the growing public demand for public services, local governments are caught in the dilemma of " collaborative inertia" in the provision of 
public services, and the initiative of collaborative development only remains at the level of declaration, and it is difficult to break through the rigid administrative boundaries without substantial progress. Under the service-oriented government model, the factors affecting the motivation of inter-governmental cooperation in the provision of public services are complex, but the ultimate factor is the interest factor, so it is imperative to take effective measures to enhance the motivation of inter-governmental cooperation in the provision of public services and turn it into practical action.

\section{RESEARCH REVIEW}

\subsection{A REVIEW OF RESEARCH ON INTER- GOVERNMENTAL RELATIONS}

\subsubsection{Foreign research review}

Foreign research on inter-governmental relations began with the financial crisis in the U.S. In the 1930s, in the face of the Great Depression, the U.S. government faced many social problems that were difficult to solve alone, and the federal government adopted an active policy to strengthen the synergy between the states and work together to deal with social panic.In 1937, Clyde F. Snider first introduced the term "inter-governmental relations" in his book Rural and Urban Government in 1935-1936. Since then, intergovernmental relations have gradually entered the field of scholarly research and have received increasing attention from academics, especially those in public administration[1].Before1980, foreign scholars' research on inter-governmental relations was mainly focused on the vertical relationship between federal and local governments. Since the 1980s, the relationship between governments in Western countries has become more complex than a single form. This has driven the study of intergovernmental relations to deepen and gradually systematize. In response to the complexity and deinstitutionalization of public service problems, Western scholars have introduced governance theory to analyze the relationship between government and market, government and civil society from the perspectives of public administration relations and interest relations, and inter-governmental governance has gradually become a new trend in the study of inter-governmental relations. At the level of the governance process, a new model of cooperation between the central and local governments, between local governments, and between local governments and non-governmental organizations is formed [2].

\subsubsection{Chinese research review}

Domestic scholars' research mainly focuses on the connotation, types, dilemmas, countermeasures, and inter-governmental interests, and have achieved fruitful results in the study of inter-governmental relations.

\subsubsection{A study on the connotation of intergovernmental relations}

According to Shangli Lin, a professor at Fudan University, intergovernmental relations mainly refer to "inter-governmental and inter-regional relations at all levels, including vertical relations between the central government and local governments, local intergovernmental relations at all levels, and horizontal interregional relations" [3].Professor Qingkui Xie, a professor at Peking University, was the first to explicitly use the concept of "inter-governmental relations" in his article A Study of Inter-governmental Relations in the Chinese Government, stating that "inter-governmental relations are relations between governments, which include relations between the central government and local governments, between local governments, between government departments, and between regional governments [4]. "

\subsubsection{A study on the types of intergovernmental relations}

The scholarly community has generalized intergovernmental relations into horizontal, vertical, and networked relations in the traditional sense, as well as oblique intergovernmental relations. In addition to the influence of foreign research theories and methodologies, the real needs of domestic development have led to a shift in the focus of research on intergovernmental relations in China. before the 1990s, research on inter-governmental relations focused on the vertical relationship between the central government and the local government, while after the 1990s it shifted to the horizontal relationship between local governments [5].

\subsubsection{A study on the dilemma of intergovernmental cooperation}

Scholars Bing Sun pointed out that the promotion game among local governments can hinder the development of cooperation [6]. Scholars Chaoshuang Long and Xiaozeng Wang pointed out that there are many obstacles in the implementation of the system of local government cross-regional cooperation, mainly in two aspects of system implementation alienation and system supervision, and proposed to solve the problem of cross-regional cooperation at the ideological, organizational and institutional levels of local governments [7].Scholars Manyun Cao and Xingyu Qi pointed out that local protectionism formed by historical and regional differences, as well as the core of local governments in dealing with inter-governmental relations is their own interests, resulting in a serious 
lack of conceptual awareness of cooperation among local governments [8].

\subsubsection{A study of countermeasures to the dilemma of inter-governmental cooperation}

Chinese public management scholars' research on the response to intergovernmental cooperation is mainly analyzed by combining the cases of Yangtze River Delta, Pearl River Delta, Beijing-Tianjin-Hebei and other economic cooperation regions. Faced with the dilemmas and problems of local inter-governmental cooperation, scholar Lan Cai analyzed the dilemmas of local government cooperation in Chang-Zhu-Tan transit integration by applying the cooperative governance framework. And she proposed countermeasures to deepen government cooperation from three perspectives: policy arena, political authority, and participation network [9].Scholars Yan Song, Peng Chen, and Ming Zhang argue that strengthening environmental supervision and constraint mechanisms, rational design of existing government incentives as well as improving the environmental transfer payment system between regions and between central and local governments can promote the benign conversion of economic performance to environmental performance [10].

\subsubsection{A study on the interest of intergovernmental relations}

According to Qingkui Xie, inter-governmental relations are the real relationships formed by public power in governmental institutions, which are manifested as inter-governmental relations that are intertwined in a vertical and horizontal manner, and which are centered on interest relations. Intergovernmental relations include both commandsubmission relations and interactive relations of competition and cooperation. The connotation of intergovernmental relations should first be interest relations, then power relations, financial relations, and administrative relations. The former determines the latter three, and the latter three are the manifestation of the former [4]. According to Zuyun Liu, the core of inter-governmental governance is the interest mechanism with "discrete power". Nowadays, the existence of local government interests and the local interests they represent is an indisputable fact. Therefore, the establishment of an incentive mechanism to balance interests and interests in order to resolve the "excessive dispersion" of local interests is one of the themes of inter-governmental governance [11]. According to Jingen Zhang, domestic intergovernmental relations are a kind of interest relationship in which interests are played and power interacts between multilateral multi-level governments within a sovereign state [12].

\subsection{A REVIEW OF RESEARCH ON COOPERATIVE INERTIA}

\subsubsection{Foreign research review}

Foreign scholars' research on "collaborative inertia" has focused on the theory of collaborative advantage. In order to unveil the dilemma of human collaboration and build a more macro and systematic theoretical framework that can cover multiple factors, a kind of "Collaborative Advantage Theory" , which focuses on the study of "collaborative inertia", has emerged after the collective action theory and has become an important tool to reveal the dilemma of collective action in society with its open, holistic and pluralistic theoretical characteristics [13].The theory of collaborative advantage suggests that in the context of the increasing complexity of modern public affairs, collaborative behavior is common in human societies because of the need to gain a "collaborative advantage". However, the theory also makes clear that there is ample evidence to suggest that collaborative advantage is not as easy to achieve as one might think, and that Collaborative Inertia often occurs, often leading to the failure of collaborative behavior in practice. Thus, the two opposing concepts of " collaborative advantage" and " collaborative inertia" form the core logical argument for the theory of collaborative advantage: the former refers to the creation of collaborative effectiveness through joint work, while the latter refers to the tendency of collaborative activities to produce highly inefficient output results or to create a large number of intolerable conflicts [14].Among the themes of collaborative practice, own observations, policy research, and theoretical findings of other researchers continue to expand on these themes, but in general the core revolves around five major point themes: goals, power, trust, membership structure, and leadership. Scholars C Huxham and S VangenDoing discuss the reasons for collaborative success in the article Things collaboratively: realizing the advantage or succumbing to inertia? they argue that two concepts are critical to this point. One is the collaborative advantage. To gain real advantage from collaboration, it is necessary to achieve some goal that no one organization acting alone can achieve. This concept provides a useful "guiding light" for the purpose of collaboration. The second concept, collaborative inertia, captures what often happens In practice: the output of collaborative arrangements ignores efficiency, the output is extremely slow, or the painful stories and hard grind are integral to the success achieved [15]. 


\subsubsection{Chinese research review}

The first domestic research paper mentioning "collaborative inertia" is the article Collaborative Inertia: A New Perspective on Collective Action Dilemma Analysis published by scholars Bin Lu and Taijun Jin, which points out that the theory of collaborative advantage, with the important content of collaborative inertia, not only opens up a new channel for theoretical development, but also provides a more reliable theoretical tool to reveal the current collective action dilemma in China. As a new trend of public affairs governance and a new development of collective action, collaborative governance needs to effectively overcome the drawbacks of collaborative inertia on the basis of institutional construction and improvement, in order to realize the real manifestation of collaborative value [16].Scholar Yaqin Zhang published On "Collaborative Inertia" in Public Service Provision and Its Transcendence, suggesting that in public service practice, "collaborative inertia" can profoundly affect the process and success of collaboration. It manifests itself in the government's "unwillingness to collaborate" due to the "blame shifting mentality", enterprises' "unwillingness to collaborate" due to the "tendency to skim", and social organizations' "volunteer failure". Social organizations are trapped by "voluntary failure" and "cannot collaborate", and citizens are "inconvenienced to collaborate" due to their "marginal status". Only by building a cooperative public service model that is public-oriented and characterized by "equality, win-win and autonomy" can the dichotomy between the "center" and "periphery" of subjects be eliminated and the "collaborative inertia" in public service provision be overcome [13].

\subsection{RESEARCH REVIEW}

To sum up, with the continuous development of modern government, the study of inter-governmental relations has been the focus of scholars' research at home and abroad. The domestic scholars' research has been fruitful, mainly focusing on the connotation, types, dilemmas, countermeasures, and inter-governmental interests of inter-governmental relations based on foreign theories with Chinese characteristics. In the context of market economy development, the essence of horizontal inter-governmental relations is the relationship of interests, and the prerequisite for efficient inter-governmental public service provision is the coordination of inter-governmental interests. However, there is little systematic research that treats "interest" as a core element that influences the motivation of inter-governmental public service provision. The research on "collaborative inertia" in China is still in its initial stage, and most of the results focus on introducing foreign theories and proposing countermeasures based on specific fields, while the research on the subject's collaborative motivation is relatively rare. At present, there is not enough research on the issue of " collaborative inertia" in China's intergovernmental public service supply, and there is a lack of necessary explanation and analysis of the " collaborative inertia" in inter-governmental public service supply with the interest factor as the key factor. It is of certain theoretical and practical significance to study the collaborative supply of inter-governmental public services from this new entry point.

\section{ANALYSIS OF THE MECHANISMS OF "COOPERATIVE ADVANTAGE" AND "COOPERATIVE INERTIA"}

Whether it is the realization of " collaborative advantage" or the creation of " collaborative inertia", the core is the problem of collaborative motivation. The analysis of collaborative motivation in this paper is based on motivational theories, including content-based motivation theory, process-based motivation theory and behavioral reinforcement theory.

Firstly, content-based motivation theory. Motivation is based on the law of activity of behavioral dynamics system, and through various management measures to continuously stimulate people's work motivation, so that their mental processes are always kept in a state of excitement and maintain a high enthusiasm for work. The starting point and basis of motivation is need, and need is the source of motivation. The core idea of content-based motivation theory is to design the motivation measures according to the source of motivation, i.e. "need". It is represented by Maslow's Hierarchy of Needs, Herzberg's Two-Factor Theory, and McClelland's Achievement Needs Theory, etc. It mainly answers the question of what motivates employees to work.

Secondly, process-based motivation theory. The conversion of needs into motivation is also conditioned by other internal factors. Process motivation is the study of how to stimulate internal factors to influence the formation of motivation and behavior change. The goalsetting theory is one of the process-oriented motivation theories. The professor Locke found that "the reason why external stimuli have an effect on motivation is the presence of the 'goal' factor". He was the first to propose the "goal-setting theory". Based on the viewpoint of goal setting theory, goals themselves have a certain motivational effect, and by setting goals, the direction of the object's behavior is guided. People will adjust their efforts in the process of pursuing the achievement of reaching goals according to the different levels of difficulty of the goals, and the influence of goals on behavior is continuous. In addition, goal setting theory suggests that goal setting affects motivational change: The higher the clarity and the more challenging the goal, the greater the motivational effect on the object [17]. 
Thirdly, behavioral reinforcement theory. Reinforcement refers to the tendency of stimuli to have a strengthening or negative reinforcing effect on motivational strengths and behavioral tendencies. Reinforcement theory is a theory proposed by Skinner, an American psychologist and behavioral scientist. Reinforcement theory points out that the motivational effect of positive reinforcement is better than that of negative reinforcement, and that negative reinforcement may cause pessimistic and dissatisfied psychological reactions in the target, or even be counterproductive to behavioral reinforcement, so we should use more positive reinforcement and less negative reinforcement when developing management initiatives.

Therefore, the "need" dimension based on the content-based motivation theory, the "goal" dimension based on the process-based motivation theory, and the "reinforcement" dimension based on the reinforcement theory. These three dimensions are fundamental to the analysis of the realization of " collaborative advantage" and the creation of " collaborative inertia" in this paper.

\subsection{The mechanism of "Collaborative advantage" in inter-governmental public service provision}

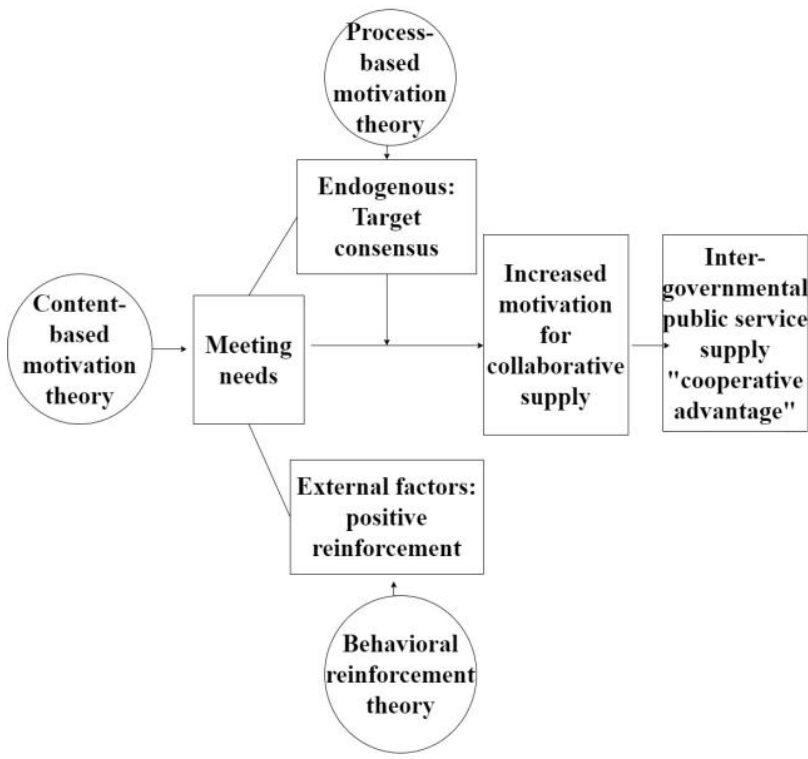

Figure 1 The generation of inter-governmental "cooperative advantage"

As shown in Figure 1, the realization of the "cooperative advantage" of inter-prefectural public service provision is the ideal state of the three dimensions combined, where local governments without administrative affiliation achieve consensus on goalsetting in the process of public service provision. This consensus may come from discussions among senior leaders or from the signing of specific interoffice agreements. Local governments and local officials meet economic, political, and social needs and achieve a relative balance of interests, and a variety of factors positively reinforce the motivation for collaborative provision.

\subsection{The mechanism of "Collaborative inertia" in inter-governmental public service provision}

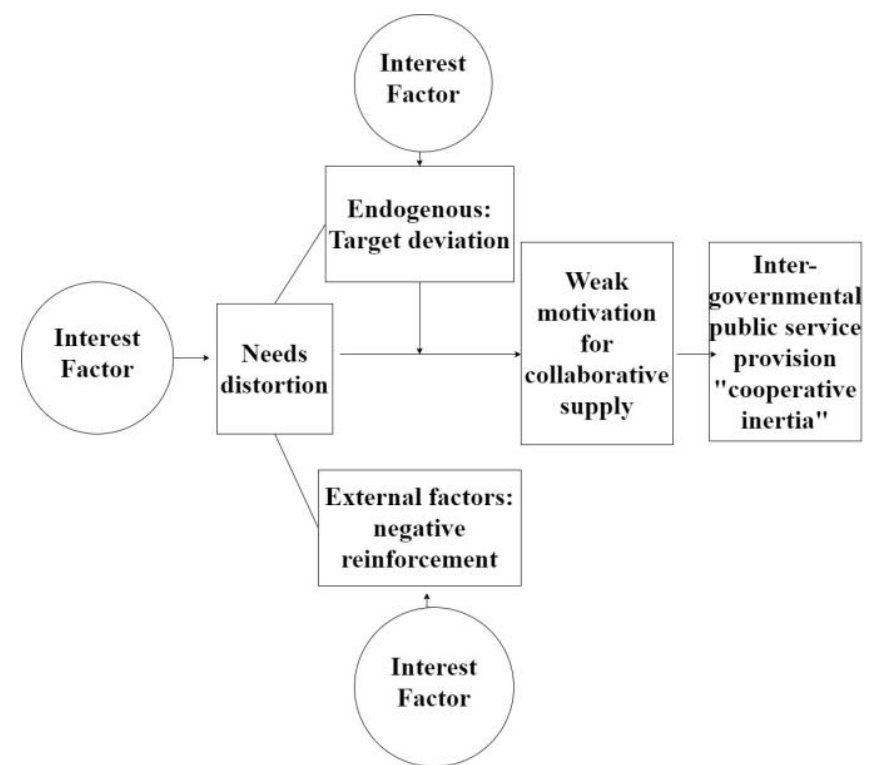

Figure 2 The generation of inter-governmental "cooperative inertia"

As shown in Figure 2, the " collaborative inertia" in inter-governmental public service provision is a real dilemma caused by the combined effect of three dimensions, and the interest factor is the key to the " collaborative inertia" in inter-governmental public service provision in China. Under the influence of the interest factor, local officials are constrained by the traditional political promotion assessment mechanism and unilaterally pursue economic growth, local financial resources are insufficient, and they are faced with interregional public service supply issues. Local governments are confined to local interests and local protection, distortion of demand, difficulty in consensus on goals, and a variety of factors have negatively reinforced the incentive for collaborative supply, and the incentive for collaborative supply is weak.

\section{A THREE-DIMENSIONAL ANALYTICAL MODEL OF "COOPERATIVE INERTIA" IN INTER- GOVERNMENTAL PUBLIC SERVICE PROVISION}

In modern society, any activity of people revolves around interests. For the purpose of this paper, local government interests are the government's interests expressed in the process of supplying cross-regional public services, which can be divided into three major 
categories: firstly, the overall interests of intergovernmental governments; secondly, the interests of individual local governments; and thirdly, the interests of government officials. The three are intertwined with economic interests, political interests, and social interests. Due to the different levels of interests of the participating parties, the conflict between economic, political and social needs, and the deviation of the overall goals of the inter-governmental government, the goals of individual local governments, and the goals of government officials, various factors negatively strengthen the motivation of inter-governmental public service cooperation and lead to the decrease of the willingness of the parties to cooperate, resulting in " collaborative inertia".

\subsection{Interest-Needs Dimension}

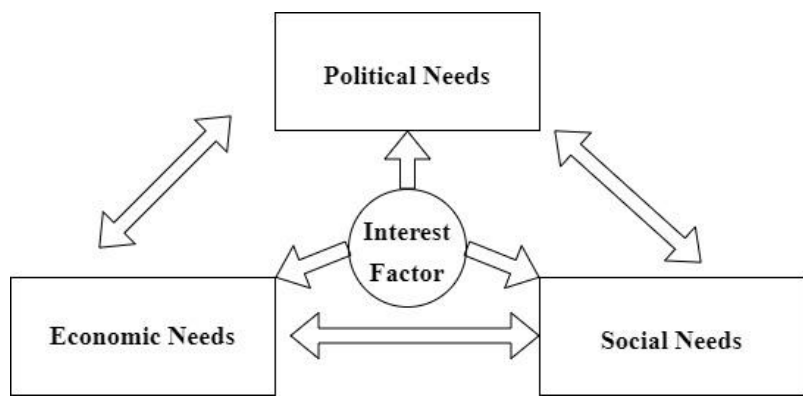

Figure 3 Interest-Needs Dimension

The competition among local governments in the inter-governmental public service provision stems from the triple identity of local governments as "economic players", "political players" and "representatives of public interests". which leads to the conflicting pursuit of "economic needs," "political needs," and "social needs". The conflict is driven by the need to maximize costs and fiscal budgets under economic interests, the need to maximize policy resources and performance under political interests, and the need to provide services efficiently and enhance public access under social interests.

According to Buchanan's public choice theory, governmental and private market actors are both selfinterested by nature, and they are "rational people" who seek to maximize their interests in the political sphere. For many local governments in China, the economic development of local governments is unbalanced and there are large disparities between regions. Local governments usually focus on economic growth and fiscal revenue in their own jurisdictions, prefer to provide public services with obvious economic benefits, and actively create local GDP growth. The noncompetitive and non-exclusive nature of public goods makes it easy for local governments to "piggyback" on inter-governmental public service provision based on their interests, enjoying the benefits of public service provision but not willing to bear the corresponding costs, hoping to pass on the costs to other local governments in the region. The distorted economic needs of local governments make the incentive for collaborative public service provision insufficient.

For a long time, the performance appraisal system of local governments and local officials in China has been mainly based on the total economic volume and growth rate of administrative districts, and the government, based on the economic-oriented view of performance, usually focuses on the tilting of policy resources and personal promotion of officials in their own jurisdictions. Local government officials engage in a "political promotion game" and are only concerned with their relative ranking to their competitors, leading to the sectoralization of public interests and the privatization of sectoral interests. In addition, the change in the leadership of local government officials can also hinder the continuation of inter-governmental coordination and supply agreements, which often leads to the dilemma of "people leaving the government". The political needs of local governments are distorted, making the incentive for collaborative provision of public services insufficient.

Local governments are caught in the pursuit of economic and political interests, neglecting social and public interests, and using short-term economic and political interests to hinder the long-term development of their regions. The public does not have a high sense of access and satisfaction in public service provision, and the social needs of local governments are distorted, resulting in insufficient motivation for intergovernmental public service provision and "collaborative inertia".

\subsection{Interest - Goals Dimension}

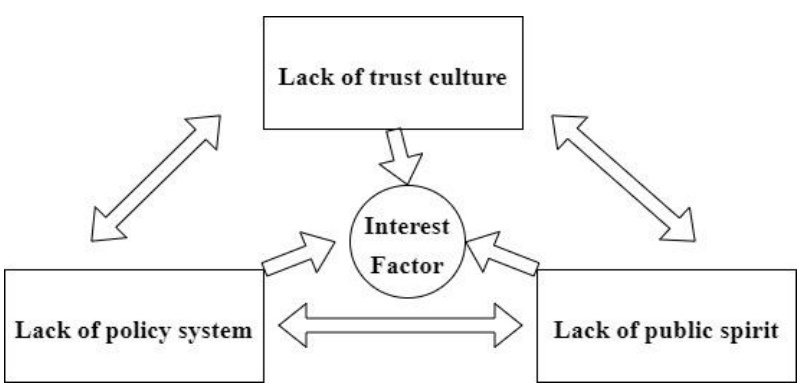

Figure 4 Interest - Goals Dimension

Local governments and government officials not only have their own unique styles of decision making and means of doing things, but also have independent local interests and personal interests to pursue. Anthony Downs, in Inside the Bureaucracy, writes that "bureaucratic organizations always issue policy recommendations from their own sphere and are constantly vigilant about the organizational sphere" [18]. 
Firstly, the goal of cooperation among inter-regional subjects of inter-governmental cooperation is to improve administrative efficiency and reduce transaction costs. The common benefits generated through cooperation are greater than the individual benefits that can be produced by local governments acting alone. Secondly, local governments, as representatives of local public interests and providers of cross-regional public services, are important representatives of local interests, and their goal is to maximize the interests of their jurisdictions. Finally, local officials have subjective initiative, and those who hold power and you can have a significant impact on local development and are important representatives of individual interests.

In fact, in an urban area, there are disparities among members of inter-governmental local governments in collaborative public service provision, whether in terms of political support, level of economic development or resource endowment. And local governments in relatively weak positions tend to have less autonomy in collaborative provision. The local disparities within the inter-governmental public service synergy provision region will be significantly widened, with each strong and beneficial local government becoming stronger, while the weak and disadvantaged local governments will be at a disadvantage. Strong local governments pursue more benefits rather than collaborate with lagging local governments. Weak and damaged local governments become passive in the process of collaborating to provide public services, and may even pass the responsibility to each other, which eventually leads to the unsustainable supply of public services.

To a large extent, local governments participate in the collaborative provision of public services from the goal of developing their own interests, without considering the goal of inter-governmental development. When the overall goal of intergovernmental "win-win cooperation" deviates from the goal of disadvantaged local governments to maximize local interests and officials to enhance their personal performance, local governments adopt a "non-zero-sum game" strategic choice, leading to the prevalence of local protectionism. The ambiguous goal setting of inter-governmental coordination in public service provision, asymmetric information and unclear positioning of rights and responsibilities among different local governments in the region eventually lead to a formal mechanism of inter-governmental coordination in public service provision, which is difficult to perform its proper function. The lack of consensus and the deviation of objectives have exacerbated the weak motivation of inter-governmental public service provision and created " collaborative inertia".

\subsection{Interest-enhancing dimension}

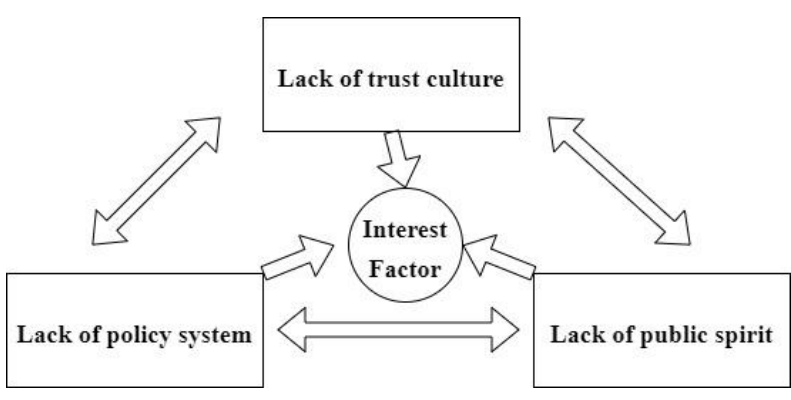

Figure 5 Interest-enhancing dimension

Under the premise of the existing conflict of interests, the lack of institutional guarantee, public spirit and trust culture further strengthen the differences in interests of subjects, weaken the motivation of intergovernmental public service supply synergy, and foster " collaborative inertia".

Institutional guarantee is the key to be able to steadily advance the inter-prefectural public service synergy supply. However, in the current development, there are some problems in the institutional guarantee, and the policy system is missing to negatively strengthen the difference of interests of the main body. So far, there are no laws and regulations specifically for inter-prefectural public service synergy, and the fair competition and win-win cooperation in interprefectural public service synergy lack a scientific and reasonable policy constraint. Although there are many inter-prefectural agreements in place, they only provide for regional affairs and do not specifically address the division of functions, powers and responsibilities of local governments in the inter-prefectural public service provision, which intensifies the unloading and shirking of responsibilities by local governments. At the meantime, the organizational mechanism of intergovernmental public service synergy supply is not perfect, the organizational structure is loose, and the resolutions reached are strongly colored by the governor, and the execution is difficult to maintain and guarantee.

Public spirit is an important guarantee of whether local governments can truly perform their service functions and safeguard public interests in the process of coordinated public service provision. As bureaucratic economists, local governments and officials, when faced with the choice between economic development and political promotion, often violate the public spirit and jeopardize the public interest by "free-riding". Strengthening administrative ethics and political ethics, and cultivating public spirit among officials from a conceptual point of view, is the only way to regulate the cooperative behavior of local governments and improve the efficiency and benefits of public service provision. 
A strong inter-governmental trust culture is the basis for efficient inter-governmental public service delivery. A stable and sustained trust relationship facilitates a consensus of interests among local governments and promotes the creation of collaborative actions. Collaborative inter-governmental public service provision is constrained by the traditional rigid administrative regions and compartmentalized administrative system, and information communication and sharing channels are not smooth. The lack of trust culture negatively reinforces the difference of subject interests. Currently, international cooperation in China is mainly carried out in the form of coordination meetings and forums, such as the "Shanghai-SuzhouZhejiang Economic Cooperation and Development Symposium" and the "Yangtze River Delta Coordination Council". However, the traditional way of government information exchange, which is mostly in the form of administrative correspondence, has not changed, and due to the lack of trust culture, the phenomenon of withholding favorable policy information from each other is also common.

\section{CONCLUSION}

Interests are the starting and ending points of social relationships. In the governance of public problems and the collaborative provision of public services, the conflicts among interest subjects are becoming more and more complex and common, and the analysis of interests has become more and more important. In this paper, we start from the key factor that affects the motivation of local governments to cooperate in horizontal public service supply subjects: the interest factor. In addition, a three-dimensional model of "collaborative inertia" is constructed based on motivational incentive theory to investigate the causes of "collaborative inertia" in inter-prefectural public service supply.

From the perspective of interest-needs dimension, the " collaborative inertia" arises because of the "economic needs", "political needs" and "social needs" distortion of local governments and local officials driven by interests. In order to alleviate the "collaborative inertia", we must first improve the interest coordination mechanism, create a good government organization atmosphere, innovate the interest compensation mechanism, and mobilize the enthusiasm of local governments to cooperate; secondly, innovate the incentive compatibility mechanism, reshape the performance assessment and appointment and dismissal of officials, establish a sound supervision and punishment mechanism, strengthen the supervision of superiors and internal supervision, and encourage social supervision.Finally, establish a public-oriented action concept, and take the maximization of public interest as the mission.
From the perspective of interest-goals dimension, the" collaborative inertia" arises because of the deviation of the overall inter-governmental goals, individual local government goals and personal goals of local officials driven by interests. In order to alleviate the " collaborative inertia", it is necessary to firstly establish an inter-governmental public service supply information system and realize the interconnection between local governments. Secondly, it is necessary to enhance the awareness of inter-governmental collaboration, improve the inter-governmental coordination and organization mechanism, and clarify the division of governmental powers and responsibilities. Finally, sound relevant laws and regulations to give legitimacy to the collaborative supply agreements and cultivate the value goal of overall regional interests.

From the interest-reinforcement dimension, the " collaborative inertia" arises due to the lack of policy system, public spirit, and trust culture, which further weaken the motivation of collaborative supply by reinforcing the interest differences of intergovernmental collaborative supply subjects. In order to relieve the "collaborative inertia", we must firstly, improve the supporting policies and regulations and reconstruct a reasonable local interest structure. Secondly, we must establish a multi-faceted demand evaluation mechanism, cultivate public spirit and build an atmosphere of public opinion for collaborative supply. Finally, strengthen communication and exchange among local governments, establish a sound credit system, and drive collaborative supply with a culture of trust.

\section{REFERENCES}

[1] Hongshan Yang, Theory of Intergovernmental Relations [M]. Beijing: China Social Science Press, 2005.

[2] Mengshi Zhang, A review of the study of intergovernmental relations: the formation system, participating subjects and specific processes[J]. Journal of Party School of Harbin Municipal Committee, 2017(03): 48-54.

[3] Shangli Lin, Domestic Intergovernmental Relations [M]. Hangzhou: Zhejiang People's Publishing House, 1998

[4] Qingkui Xie, A study of inter-governmental relations in Chinese government[J]. Journal of Peking University (Philosophy and Social Science Edition), 2000(01):26-34.

[5] Jingen Zhang, Intergovernmental governance: New trends in the study of inter-governmental relations in contemporary China[J]. Academic Research, 2013(2) 
[6] Bing Sun, Study on the development of local government cooperation in China in the context of promotion game $[\mathrm{J}]$. Nankai Journal (Philosophy and Social Science Edition), 2013(2):23-30.

[7] Chaoshuang Long, Xiaozeng Wang, Research on the dynamics mechanism of cooperation among local governments in China[J]. China Administration,2007(6):65-68.

[8] Mangyun Cao, Xingyu Qi, A preliminary study on information asymmetry in cross-regional cooperation governance among local governments [J]. Journal of Leshan Normal College, 2006(1): 100-103.

[9] Lan Cai, Yun Feng, Analysis of the fit between ANSELL cooperative governance framework and China's regional cooperation--Take Chang-ZhuTan bus integration as an example[J]. Academic Research, 2014(09): 58-64+159.

[10] Yan Song, Sai Chen, Ming Zhang, Local government heterogeneity and regional environmental cooperative governance-an evolutionary game analysis based on Chinese style decentralization $[\mathrm{J}]$. China Management Science, 2020, 28(01): 201-211.

[11] Zuyun Liu, Intergovernmental relations: cooperative games and inter-governmental governance[J]. Xuehai, 2007(01):79-87.

[12] Jingen Zhang, Introduction to contemporary intergovernmental relations in China [M]. Beijing: Social Science Literature Press, 2009.

[13] Yaqin Zhang, On the "collaborative inertia" in public service provision and its transcendence[J]. Xuehai, 2017(06):25-31.

[14] Osborne, translated by Guoxian Bao, New Public Governance? --New perspectives on the theory and practice of public governance [M]. Beijing: Science Press.2016.

[15] C. Huxham, S. Vangen. Doing things collaboratively: realizing the advantage or succumbing to inertia?[J]. IEEE Engineering Management Review,2004,32(4).

[16]] Bin Lu, Taijun Jin, Collaborative inertia: A new perspective on the analysis of collective action dilemmas[J].Social Science Research,2015(04):7278 .

[17] Zihan Song, Research on the improvement of participation dynamics of public crisis governance in China [D]. University of Electronic Science and Technology of China, 2016.
[18] Anthony Downes, translated by Xiaocong Guo, Inside the Bureaucracy [M]. Beijing: People's University of China Press, 2006: 229-230. 